\title{
Influência da idade e gênero na capacidade de crianças em incorporar hábitos de saúde bucal após atividades educativas nas escolas
}

\author{
Influence of age and sex on children's ability to incorporate oral health habits after \\ educational activities in schools
}

\author{
Tauane Ramaldes Martins ${ }^{1}$ \\ Orcid: orcid.org//0000-0002-0817-0018 \\ Ana Paula Ribeiro ${ }^{3}$ \\ Orcid: orcid.org/0000-0002-1061-3789
}

\author{
Yara Juliano ${ }^{2}$ \\ Orcid: orcid.org/0000-0002-8391-075X
}
Christiane Carvalho Fonseca Ribeiro ${ }^{4}$
Orcid: orcid.org/0000-0002-3115-2037

\author{
Neil Ferreira Novo 5 \\ Orcid: orcid.org/0000-0001-7903-8156
}

\begin{abstract}
Resumo
Introdução: A promoção da saúde bucal é um dos pilares de estratégias preventivas no surgimento de alterações dentárias nas crianças escolares, visto sua maior facilidade na incorporação de hábitos saudáveis e higiênicos nas escolas. Porém, até o momento pouco se compreende sobre a influência da idade e gênero sobre a capacidade das crianças na compreensão, melhora e aderência à saúde bucal após ações educativas nas escolas. Objetivo: Verificar a influência de diferentes faixas etárias e gênero na capacidade das crianças em incorporar hábitos de saúde bucal após atividades educativas nas escolas. Casuística e Métodos: Foram avaliadas 173 crianças de uma Escola Estadual pública pertencente à região Sul da subprefeitura da Capela do Socorro da cidade de São Paulo, os quais foram divididas, de acordo com a faixa etária, em três grupos: 6-7 anos $(n=62)$; 8-9 anos $(n=47)$ e 10-11 anos $(\mathrm{n}=27)$. Em seguida, foram aplicados questionários aos responsáveis legais pelas crianças, compondo os seguintes tópicos: dados demográficos e socioeconômicos, hábitos alimentares e de higiene bucal, bem como o Índice de Higiene Oral Simplificado (SOHI). A estratégia educativa foi realizada por meio de palestras, teatros, histórias sobre escovação e higiene bucal. Após duas e quatro semanas da ação educativa todos os dados foram reavaliados. Resultados: Houve uma diferença significativa entre a faixa etária dos 6 a 7 anos de idade em relação aos grupos de 8 a 9anos e de 10 a 11 anos, sendo os mais jovens mais responsivos e efetivos na saúde e higiene bucal, após duas e quatro semanas da ação educativa na escola. Em relação aos gêneros, não se observou diferenças intra e inter grupos. Conclusão: Crianças escolares de diferentes faixas etárias: 6 a 7, 8 a 9 e 10 a 11 anos, apresentaram melhoras efetivas de saúde bucal após duas e quatro semanas da ação educativa na escola, independente do gênero em cada faixa etária. A incorporação do hábito de saúde bucal foi melhor nos mais jovens com faixa etária dos 6 aos 7 anos de idade.
\end{abstract}

Palavras-chave: oral health, children, school

\footnotetext{
${ }^{1}$ Faculdade de Odontologia, Universidade Santo Amaro, Departamento de Pós-Graduação em Ciências da Saúde, São Paulo, Brasil. E-mail: tauane_odonto@hotmail.com

${ }^{2}$ Universidade Santo Amaro, Departamento de Pós-Graduação, Mestrado em Ciências da Saúde, São Paulo, Brasil. E-mail: yjuliano@prof.unisa.br

${ }^{3}$ Universidade Santo Amaro, Laboratório de Biomecânica e Reabilitação Musculoesquelética-LaBiREM, Departamento de Pós-Graduação, Mestrado em Ciências da Saúde, São Paulo / SP, Brasil. E-mail: anapribeiro@prof.unisa.br

4 Faculdade de Odontologia, Universidade José do Rosário Vellano, Alfenas / Minas Gerais, Brasil. E-mail: chrisfonseca41@gmail.com

${ }^{5}$ Universidade Santo Amaro, Departamento de Pós-Graduação, Mestrado em Ciências da Saúde, São Paulo, Brasil. E-mail: nnovo@prof.unisa.br
} 


\begin{abstract}
Introduction: The promotion of oral health is one of the pillars of preventive strategies in the emergence of dental changes in schoolchildren, as it is easier to incorporate health and hygienic habits in schools. However, to date, little is understood about the influence of age and sex on children's ability to understand, improve, and adhere to oral health habits after educational actions in schools. Objective: To verify the influence of different age groups and sex on children's ability to incorporate oral health habits after educational activities in schools. Materials and Methods: 173 children from a public State school, located in the South Region of the Capela do Socorro subprefecture of the city of São Paulo were evaluated and divided according to age into three groups: 67 years $(n=62), 8-9$ years $(n=47)$, and $10-11$ years $(n=27)$. Questionnaires were applied comprising the following topics: demographic and socioeconomic data, eating habits and oral hygiene, as well as the Simplified Oral Hygiene Index (SOHI). The educational strategy was performed with lectures, theater, and stories about brushing and oral hygiene. After two and four weeks of the educational action all data were reevaluated. Results: There was a significant difference between the 6 to 7 year age group compared to the 8 to 9 year and 10 to 11 year groups, with the youngest being more responsive and effective in oral health and hygiene, after two and four weeks of educational action at school. Regarding sex, there were no differences within and between groups. Conclusion: Schoolchildren of different age groups: 6 to 7,8 to 9 , and 10 to 11 years, showed effective improvements in oral health after two and four weeks of an educational action at school, regardless of sex. The incorporation of oral health habits was better in the 6 to 7 year age group.
\end{abstract}

Keywords: oral health, children, school, age

\section{Introdução}

Ações educativas de saúde bucal são realizadas no ambiente escolar brasileiro desde o início do século XX, em 1912, quando foi implantado o Programa de Saúde Bucal para Escolares na cidade de São Paulo ${ }^{1}$ O ambiente escolar estimula o conhecimento crítico e a incorporação de hábitos saudáveis no cuidado bucal das crianças, podendo chegar até em repercussões em seus familiares ${ }^{2,3}$.

Muito da importância da saúde bucal em escolares vem da alta prevalência da doença cárie e periodontal que atingem crianças e adolescentes nos diferentes ciclos de vida, cuja evolução resulta em perdas de elementos dentários, sendo considerada um problema de saúde pública que necessita de programas preventivos por meio de orientação e motivação do cuidado de higiene bucal para prevenção das doenças dentárias em crianças e altos custos com tratamentos especializados ${ }^{4,5,6}$. odontológicos

A literatura revela que ações educativas de higiene bucal com duração de 15 minutos em crianças pré-escolares de 4 a 6 anos de idade, após curto período de quatro semanas, atingiram melhoras significativas na prática de higiene bucal e limpeza das mãos e unhas, sendo que as meninas apresentaram maior efetividade quando comparado aos meninos ${ }^{6}$. Outro estudo realizado por Ferrazzano et al., $(2008)^{4}$, também em crianças, verificou uma melhora expressiva na saúde bucal após uma semana das ações educativas de saúde bucal, sendo maior nas meninas, mas se manteve baixo após três meses. Os autores concluíram que foi um método efetivo para o estabelecimento de bons hábitos de saúde bucal pra crianças.

Ainda nessa linha de raciocínio, Ribeiro, et al., (2009) ${ }^{2}$ avaliou um método educativo preventivo e de motivação aplicado a crianças do $5^{\circ}$ ano do ensino fundamental da rede pública da cidade de Ribeirão Preto, SP. Trinta e três escolares com idades entre 9 e 11 anos responderam um questionário de caracterização da amostra e conhecimento e hábitos de saúde bucal em dois momentos, antes e após atividade educativa. Os autores concluíram que o programa educativo preventivo foi capaz de despertar o interesse das crianças além de promover um desenvolvimento intelectual e contribuir na motivação dos hábitos de higiene, porém para que a atividade seja efetiva, os programas de 
prevenção devem ser feitos periodicamente. Farias et al., (2009) $)^{5}$ ao determinarem o impacto da educação em saúde e higiene bucal em 257 escolares de 7 a 15 anos de idade verificaram, antes e após 4 meses da ação educativa em saúde bucal, os índices, CPO-S (Cariado Perdidos e Obturados Superfície), o Índice de Placa Visível (IPV) e o Índice de sangramento gengival. O nível de informação sobre saúde bucal dos escolares não teve relação com os resultados dos índices avaliados, ou seja, escolares mais informados nem sempre praticam a melhor higiene bucal. Outros estudos verificaram que crianças escolares entre 7 a 10 anos de idade que receberam ações educativas por meio de teatro, história, palestras e gincanas relacionados à saúde bucal melhoraram efetivamente seus hábitos bucais e o conhecimento sobre a temática abordada ${ }^{8,9}$.

Apesar das ações educativas de saúde bucal promoverem benefícios em crianças escolares, ainda permanece baixa a conscientização das crianças na realização da saúde bucal, fato esse que pode ser melhor compreendido pelas diferentes faixas etárias e do gênero, visto que os estudos não separam em grupos de diferentes faixas etárias e os gêneros. A escola exerce papel fundamental na orientação e na formação das crianças, o que se estende ao ambiente familiar. A instituição de atividades educativopreventivas para saúde bucal na escola, além de ser simples, de fácil aplicação e baixo custo, é de grande valor na prevenção de doenças bucais biolme-dependentes ${ }^{10}$. Assim, o objetivo deste estudo foi verificar a influência de diferentes faixas etárias e gênero na capacidade das crianças em incorporar hábitos de saúde bucal após atividades educativas nas escolas.

\section{Materiais e Métodos}

\section{Amostra e tipo de estudo}

A amostra foi composta por crianças escolares de 6 a 11 anos de idade, matriculadas em uma Escola Estadual pública pertencente à região Sul da subprefeitura da Capela do Socorro da cidade de São Paulo (SP). Cento e setenta e três crianças participaram do estudo, sendo alocadas em três grupos, segundo a sua faixa etária: crianças de 6-7 anos $(\mathrm{n}=62)$; crianças 8-9 anos $(n=47)$ e crianças de 1011 anos $(\mathrm{n}=27)$, que participaram de atividades educativo-preventivas (AEP). A partir de uma listagem única das crianças por faixa etária, as mesmas foram designadas aleatoriamente aos grupos por sorteio.

Todos os dados das crianças foram coletados após aprovação do Comitê de Ética em Pesquisa Local (número: 2015699) e após o responsável pela criança concordar e assinar o Termo de consentimento Livre e Esclarecido-TCLE para realização do estudo, respeitando os princípios éticos regidos pela resolução 466/12 do Conselho Nacional de Ética em Pesquisa-CONEP. Os dados coletados foram avaliados inicialmente e após duas e quatro semanas da ação educativa em saúde bucal. Vale ressaltar que os questionários foram aplicados aos responsáveis legais pelas crianças.

\section{Delineamento da pesquisa}

Trata-se de um estudo prospectivo analítico, por meio de intervenções educativas sobre saúde bucal em crianças escolares.

\section{Critérios de Inclusão e Exclusão}

Os critérios de inclusão de crianças no estudo foram ausência de doenças sistêmicas e do uso de medicamentos. As doenças sistêmicas consideradas como critérios de inclusão foram: doenças infecciosas agudas da infância que promovem uma queda da resistência orgânica, como gripe, catapora, rubéola e qualquer outra doença infecciosa que se fizesse presente; febre com causa conhecida ou não; discrasias sanguíneas (anemia e hemofilia); diabetes mellitus; cardiopatias; febre reumática; e doenças renais. Quanto 
aos medicamentos, foram considerados os que pudessem alterar o fluxo salivar como anti-histamínicos (para alergias); sedativos (para tosse); antiespasmódicos (para alívio de dores); medicamentos para asma e medicamentos contendo sacarose como xaropes e tônicos ${ }^{11}$.

\section{Procedimentos}

Dados socioeconômicos e demográficos, de saúde geral e hábitos de saúde bucal das crianças foram avaliadas por meio dos seus responsáveis legais, os quais assinaram o TCLE. Para tanto, foram realizadas as avaliações da placa aderida aos dentes dos escolares, as quais foram pigmentadas com fucsina básica a $0,7 \%$ (Eviplac- Solução Evidenciadora de Placa Bacteriana- Biodinâmica Química e Farmacêutica LTDA; Reg ANVISA:10298550044) e aplicada na superfície dental com algodão em hastes flexíveis. Os exames foram realizados em ambientes claros e arejados com iluminação natural pela pesquisadora principal. Após isso, foi avaliado o Índice de Higiene Oral Simplificado (IHOS), onde são verificados: a face vestibular dos dentes 16 (primeiro molar superior direito permanente), 11 (incisivo central superior direito permanente), 26 (primeiro molar superior esquerdo permanente) e 31 (incisivo central inferior esquerdo permanente) e face lingual dos dentes 36 (primeiro molar inferior esquerdo permanente) e 46 (primeiro molar inferior direito permanente) - na ausência desses dentes são utilizados os dentes decíduos correspondentes ou o dente vizinho como referência. Cada superfície recebe um código de 0 a 3 onde zero significa que a face não apresenta biofilme, código um, o biofilme está presente no terço cervical, código dois, o biofilme está presente no terço cervical e médio e código três, o biofilme cobre os três terços da face dental que varia de 0 a 2 (sem biofilme até a sua presença). Esses valores são somados e divididos pela quantidade de dentes examinados, obtendo a média que pode significar: satisfatório, regular e insatisfatório.

\section{Ação educativa em Saúde Bucal}

Atividade educativa: Após

avaliação inicial, a atividade educativa em saúde bucal foi realizada junto às crianças dos diferentes grupos de faixa etária. Os recursos didáticos usados nas atividades educativas foram brincadeiras interativas, macromodelos, escova e creme dental, cartazes e palestras. Essa atividade teve duração de 60 minutos. As atividades deram ênfase a temas como cárie dentária, gengivite, escovação com creme dental, uso de fio dental e relação da dieta com cárie dentária. Linguagem clara e objetiva foi utilizada com o intuito de tornar as crianças atentas, receptivas e motivadas à aquisição de novos hábitos para saúde bucal. Ação preventiva: As crianças receberam um kit contendo escova dental, creme dental fluoretado (1.100 ppmF) e um cartilha de orientações sobre saúde bucal. Escovação supervisionada foi realizada individualmente $\mathrm{e}$ as crianças foram orientadas para expectorar o creme dental. Essas ações educativas e a técnica de escovação foram com base nos estudos realizados por Barreto et al. $(2013)^{11} \mathrm{e}$ Frencken et al. $(2001)^{13}$.

\section{Análise estatística}

Os dados coletados foram submetidos à análise de variância de Friedman para comparar os valores do Índice de Higiene Oral Simplificado (IHOS) entre os grupos nos momentos pré, após duas e quatro semanas da ação educativa. Essa análise foi aplicada, separadamente, para o gênero feminino ou masculino dos escolares para cada um dos grupos etários. O Teste de Mann Whitney para comparar os grupos de escolares do gênero feminino ou masculino, em relação aos índices observados em cada um dos momentos de avaliação, em separado para cada grupo etário. E a análise de variância de Kruskal-Wallis para comparar os grupos em relação às diferenças entre os valores do 
IHOS, nos momentos pré-orientação e após 4 semanas. Essa análise foi feita, em separado para os alunos do gênero feminino ou masculino. O nível de significância adotado foi de $5 \%$.

\section{Resultados}

Na tabela 1, pode-se verificar que não houve diferença quando comparados os gêneros feminino e masculino, exceto pelo grupo etário de 6 a 7 anos no período préorientação, onde os meninos apresentaram índices mais altos que as meninas, significando que possuem maior quantidade de biofilme dental. Na análise de Variância de Friedman (Tabela 1) pode-se observar que houve melhora da higienização após ação educativa e que essa se manteve no período de 4 semanas no grupo 6 a 7 anos e 10 a 11 anos, em ambos os gêneros (feminino e masculino) e no grupo de 8 a 9 anos foi observado apenas no gênero feminino. Porém, um achado importante nesse mesmo grupo de 8 a 9 anos foi observar que no período de quatro semanas os índices já estavam semelhantes ao coletado antes da intervenção.

Tabela 1 - Escolares do ensino fundamental I, segundo gênero e grupo etário em relação ao Índice de Higiene Oral Simplificado (IHOS).

\begin{tabular}{|c|c|c|c|c|c|c|c|c|}
\hline \multirow[t]{3}{*}{$\begin{array}{l}\text { Idade } \\
(\text { anos })\end{array}$} & \multicolumn{6}{|c|}{$\begin{array}{c}\text { Teste de Mann Whitney } \\
\text { (feminino - F x masculino - M) }\end{array}$} & \multicolumn{2}{|c|}{$\begin{array}{c}\text { Análise de Variância de } \\
\text { Friedman } \\
\text { (pré x } 2 \text { semanas x } 4 \text { semanas) }\end{array}$} \\
\hline & \multicolumn{2}{|c|}{ Pré } & \multicolumn{2}{|c|}{ Pós-2 semanas } & \multicolumn{2}{|c|}{ Pós-4 semanas } & \multirow{4}{*}{$\begin{array}{l}F \\
x^{2} r=23,16 \\
p<0,0001 \\
2 x 4<\text { pre }\end{array}$} & \multirow[b]{3}{*}{$\mathrm{p}<0,0001$} \\
\hline & $\mathrm{F}$ & $\mathrm{M}$ & $\mathrm{F}$ & M & $\mathrm{F}$ & M & & \\
\hline \multirow[t]{2}{*}{$6-7$} & 1,6 & 1,9 & 0,89 & 1,13 & 1,09 & 1,25 & & \\
\hline & \multicolumn{2}{|c|}{$\begin{array}{l}0,010^{*} \\
2,5628(\mathrm{~F}<\mathrm{M})\end{array}$} & \multicolumn{2}{|c|}{$\begin{array}{l}0,211 \\
1,249\end{array}$} & \multicolumn{2}{|c|}{$\begin{array}{c}0,102 \\
1,6314\end{array}$} & & $2 \times 4<$ pre \\
\hline \multirow[t]{2}{*}{$8-9$} & 1,4 & 1,5 & 0,92 & 0,91 & 1,08 & 1,23 & \multirow{2}{*}{$\begin{array}{l}\mathrm{x}^{2} \mathrm{r}=23,13 \\
\mathrm{p}<0,0001 \\
2 \mathrm{x} 4<\text { pre }\end{array}$} & \multirow{2}{*}{$\begin{array}{l}x^{2} r=15,86 \\
p<0,0001 \\
2<4\end{array}$} \\
\hline & \multicolumn{2}{|c|}{$\begin{array}{c}0,504 \\
0,6673\end{array}$} & & & \multicolumn{2}{|c|}{$\begin{array}{c}0,208 \\
1,2578\end{array}$} & & \\
\hline $10-11$ & 1,3 & 1,4 & 1,02 & 1,13 & 1,24 & 1,44 & $\begin{array}{l}x^{2} r=13,46 \\
p<0,0012\end{array}$ & $\begin{array}{l}x^{2} r=15,86 \\
p<0,0105\end{array}$ \\
\hline $\begin{array}{l}\mathrm{P} \\
\mathrm{z}\end{array}$ & \multicolumn{2}{|c|}{$\begin{array}{c}0,364 \\
0,9077\end{array}$} & \multicolumn{2}{|c|}{$\begin{array}{c}0,498 \\
0,6769\end{array}$} & \multicolumn{2}{|c|}{$\begin{array}{c}0,254 \\
1,1385\end{array}$} & $\begin{array}{l}2 \times 4<\text { pre } \\
2<4\end{array}$ & $\begin{array}{l}2 \times 4<\text { pre } \\
2<4\end{array}$ \\
\hline
\end{tabular}

No teste de Variância de KruskalWallis (Tabela 1), pode-se perceber que o gênero feminino apresentou diferença estatística entre os grupos: 6 a 7 anos e 10 a 11 anos $(p<0.05)$, no qual a menor faixa etária apresentou aumento da melhora da saúde bucal em relação à faixa etária superior (10 a 11 anos). Para o gênero masculino foi encontrada diferença estatística entre os grupos: 6 a 7 anos e 8 a 9 anos $(p<0.05)$ e 10 a 11 anos $(p<0.05)$, novamente, o grupo mais jovem apresentou melhoria significativa quando comparado aos grupos mais velhos. Independente do gênero, o grupo mais jovem apresentou melhores resultados do Índice de Higiene Oral Simplificado (IHOS) que reflete um maior aprendizado da intervenção de educação em saúde bucal.

Quando se observa a tabela 2, é possível verificar nenhuma diferença estatística entre os gêneros dentro dos diferentes grupos etários. Já no período de 4 semanas (Tabela 3), não existe diferença estatística entre os gêneros dentro dos grupos etários, porém quando comparados, 
independente do gênero, nota-se que houve melhora significativa nos escolares de 6 a 7

anos.

Tabela 2 - Escolares segundo os gêneros e os grupos etários em relação ao número e porcentagem de melhora do Índice de Higiene Oral Simplificado nos períodos pré-ação educativa e após 2 semanas

\begin{tabular}{|c|c|c|c|c|c|}
\hline Idade & Melhorou (\%) & Não & lorou & Teste $C$ & ladrado \\
\hline \multirow[b]{2}{*}{$6-7$} & $\mathbf{M}$ & $\mathbf{F}$ & $\mathbf{M}$ & $\mathbf{F}$ & $\mathbf{M}$ \\
\hline & $23(82) 39(81)$ & 5 & 9 & $x^{2}=0$, & $\mathrm{p}=0,833$ \\
\hline Total & $62(81,5)$ & \multicolumn{2}{|c|}{14} & & \\
\hline 8-9 & $29(85) 18(72)$ & 5 & 9 & \multicolumn{2}{|c|}{$x^{2}=1,57, p=0,354$} \\
\hline Total & 47 (79) & \multicolumn{2}{|c|}{14} & & \\
\hline 10-11 & $18(72) 9(69)$ & 5 & 9 & \multicolumn{2}{|c|}{$x^{2}=0,03, p=0,847$} \\
\hline Total & $27(71,1)$ & \multicolumn{2}{|c|}{14} & & \\
\hline
\end{tabular}

Tabela 3 - Escolares segundo os gêneros e os grupos etários em relação ao número e porcentagem de melhora do Índice de Higiene Oral Simplificado nos períodos pré-ação educativa e após 4 semanas

\begin{tabular}{|c|c|c|c|c|c|}
\hline Idade & Melhorou (\%) & Não n & lorou & Teste $C$ & ladrado \\
\hline \multirow[b]{2}{*}{$6-7$} & $\mathbf{M}$ & $\mathbf{F}$ & $\mathbf{M}$ & $\mathbf{F}$ & $\mathbf{M}$ \\
\hline & $24(85) 43(89)$ & 4 & 5 & $x^{2}=0,2$ & $p=0,892$ \\
\hline Total & $67(88.2)$ & \multicolumn{2}{|c|}{9} & & \\
\hline 8-9 & $22(64) 16(64)$ & \multicolumn{2}{|l|}{12} & \multicolumn{2}{|c|}{$x^{2}=0,01, p=0,826$} \\
\hline Total & $38(64,4)$ & \multicolumn{2}{|c|}{21} & & \\
\hline $10-11$ & $10(40) 7(53)$ & 15 & 6 & \multicolumn{2}{|c|}{$x^{2}=0,66, p=0,636$} \\
\hline Total & $17(44.7)$ & \multicolumn{2}{|c|}{21} & & \\
\hline
\end{tabular}

Como podemos observar a análise estatística não mostrou diferença significante entre os gêneros feminino ou masculino em todos os grupos etários, tanto no período de 2 quanto no de 4 semanas.

\section{Discussão}

A educação em saúde bucal se faz fundamental para aumentar o conhecimento e aderência às mudanças de comportamento de crianças escolares para hábitos mais saudáveis de higiene bucal. Segundo alguns autores, essas mudanças devem ser instituídas na fase inicial da vida escolar, visto que as crianças são mais receptivas e aprendem facilmente permitindo a consolidação dos saberes apreendidos ${ }^{1,2}$. Corroborando com os autores, no presente estudo, a atividade educativa associada à prevenção da saúde bucal se deu mais efetiva e responsiva nas faixas etárias menores, sendo as de 6 a 7 anos, a mais efetiva após 2 e 4 semanas da ação educativa na escola. Esse que enfatiza ainda mais o fundamental papel que a escola exerce na orientação e formação da criança como futuros cidadãos ${ }^{10}$. 
Alguns estudos na literatura têm abordado faixas etárias específicas na efetividade de ações educativas na saúde bucal de crianças escolares. Barreto et al., $(2013)^{11}$ avaliou 38 crianças de 3 a 5 anos e os resultados mostraram que uma atividade educativa - preventiva é eficaz para o contexto estudado, porém para que hábitos sejam perpetuados deve haver continuidade do programa e participação dos pais. Já Antônio et al., $(2015)^{8}$ estudaram 112 crianças de 7 a 10 anos e verificaram que logo após a intervenção de educação em saúde a história em quadrinhos foi a estratégia mais eficaz, porém, de maneira geral, os métodos educativos (palestras, teatro e macromodelos) apresentados não influenciaram no conhecimento adquirido da amostra estudada. Apesar deste estudo não ter comparado métodos de ações educativas, teve-se o cuidado de utilizar os mesmos métodos aplicados no estudo de Antônio et al. $(2015)^{8}$, e observa-se a influência da faixa etária mais jovem para maior ganho de efetividade de saúde bucal, não diferenciado entre os gêneros.

Em relação aos gêneros, neste estudo não se observou diferenças para as variáveis avaliadas, porém grande tem sido a literatura que verifica o gênero feminino com maior efetividade e facilidade de aderência e melhora na saúde bucal ${ }^{4,6}$. Talvez, a não diferença entre os gêneros observada neste estudo seja devido à padronização dos diferentes grupos de faixa etária, o qual promoveu pequenas variações entre os grupos avaliados.

Vários estudos revelam que ações educativas são capazes de promover o interesse de crianças e adolescentes ${ }^{12,13}$. Ribeiro, et al. $(2008)^{5}$ avaliou um método educativo preventivo e de motivação aplicado a crianças do $5^{\circ}$ ano do ensino fundamental da rede pública da cidade de Ribeirão Preto, SP. Trinta e três escolares com idades entre 9 e 11 anos responderam um questionário de caracterização da amostra e conhecimento e hábitos de saúde bucal em dois momentos, antes e após atividade educativa. Os autores concluíram que o programa educativo preventivo foi capaz de despertar o interesse das crianças, além de promover um desenvolvimento intelectual e contribuir na motivação dos hábitos de higiene, porém para que a atividade seja efetiva, os programas de prevenção devem ser feitos periodicamente, corroborando com os achados do presente estudo.

Já Oliveira e Martini et al. (2009) ${ }^{14}$, correlacionou a aplicação de bochechos fluoretados com o IHOS de sessenta e uma crianças e adolescentes entre 6 a 13 anos de uma escola de ensino fundamental da cidade de Nova Aurora/PR. Como resultado, os alunos de menor faixa etária apresentaram resultados piores e os bochechos com flúor não alteraram IHOS, concluindo que os bochechos devem estar associados à educação em saúde com temas como: fatores etiológicos, causas e consequências da placa bacteriana, técnicas de higienização, manutenção dos instrumentos de higiene e o risco de não se higienizar corretamente. Não foi foco deste estudo verificar os bochechos, mas diferentemente, as faixas etárias menores foram as mais responsivas para melhora da saúde bucal, independente do gênero.

Apesar deste estudo não ter avaliados a influência dos professores e familiares, a literatura revela que as ações preventivas de saúde bucal devem ser de grande importância e essencial no ambiente escolar e poderia ser reiterada pelos professores, os quais exercem papel ativo na solidificação dos hábitos de higiene bucal das crianças. Além disso, a participação dos pais é fundamental, pois reforça a atividade realizada com as crianças na escola ${ }^{15,16,17 .}$

\section{Conclusão}

Crianças escolares de diferentes faixas etárias: 6 a 7, 8 a 9 e 10 a 11 anos, apresentaram melhoras efetivas de saúde bucal após duas e quatro semanas da ação 
educativa na escola, independente do gênero em cada faixa etária. A incorporação do hábito de saúde bucal foi melhor nos mais jovens com faixa etária dos 6 aos 7 anos de idade.

\section{Referências}

1. Martins EM. Educação em saúde bucal: os desafios de uma prática. Caderno de Odontol. 1998;1(2):30-40.

2. Zanirati VF, Caldas BG, Lopes ACS, Santos LC. Pediatria Moderna. 2013; 49(2):87-93.

3. Kay EJ, Locker D. Is dental health education effective: a systematic review of current evidence. Community Dent Oral Epidemiol. 1996; 24:231-5.

4. Ferrazzano GF, Cantile T, Sangianantoni G, Ingenito A. Effectiveness of a motivation method on the oral hygiene of children. Eur J PaediatrDent. 2008;9(4):183-187.

5. Ribeiro DG, Dovigo LN, Silva, SRC. Avaliação de um método educativo em saúde bucal aplicado em escolares de ensino público. Arquivos em Odontologia. 2009;45(03):154-59.

6. Figueira TR, Leite ICG. Percepções, conhecimentos e práticas em saúde bucal de escolares. RGO. 2008;56(1):27-32.

7. De Farias IA, de Araújo Souza GC, Ferreira MA. A health education program for Brazilian public schoolchildren: the effects on dental health practice and oral health awareness. J Public Health Dent. 2009;69(4);225-30.

8. Antonio LP, Gouvêa GR,de Souza LZ, Cortellazzi KL. Avaliação de diferentes métodos educativos em saúde bucal em crianças na faixa etária de 7 a 10 anos de idade. RFO, Passo Fundo;2015;20(1);52-58.

9. de Sousa JB, Lima EMM, Bento AKM, Queiroz LGS, da Silva CHF. Saúde bucal na escola: Um estudo sobre atividades de educação em saúde para estudantes. JOAC 2017;3(1):6.

10. Martins CC, Bonanato KT, Valério DS, Moura Leite FR, Paiva SM, Vale MPP. Effectiveness of an educative techinique on knowledge acquisition on rational use of fluorine by parents. J Dent Sci. 2006;21:105-11.

11. Barreto DM, Paiva SM, Ramos-Jorge ML, Ferreira MC. Evaluation of the effectiveness of an educational- preventive activity with preschoolers: pilot study for a randomized clinical trial. ArqOdontol, 2013;49(3):113-121.

12. Moura-Leite FR, Ramos-Jorge ML, Bonanato K, Paiva SM, Vale MP, Pordeus IA. Prevalence, intensity and impacto of dental pain in 5-year-old preschool children. Oral Health Prev Dent. 2008; 6:295-301.

13. Frencken JE, Borsum-Andersson K, Makoni F, Moyana F, Mwashaenyi S, Mulder J. Effectiveness of an oral health education programme in primary schools in Zimbabwe after 3.5 years. Community Dent Oral Epidemiol. 2001; 29:253-9.

14. Oliveira BRG, Martini L. Índice de higiene oral simplificado em alunos de ensino fundamental avaliado antes do bochecho com flúor. $4^{\circ}$ Seminário Nacional Estado e Políticas Sociais. 2009;16.

15. Silveira JLGC, de Oliveira V, Padilha WWN. Evaluation of reduction of the visible plaque index and of the gum bleeding index in a program of oral health promotion for children. PesquiOdontol Bras. 2002; 16:32-42. 
16. Zanin L, Meneghim MC, Assaf AV, Cortellazzi KL, Pereira AC. Evaluation of an educational program for children with high risk of caries. J Clin Pediatr Dent. 2007; $31: 246-50$.

17. Hochstetter AS, Lombardo MJ, D'eramo L, Piovano S, Bordoni N. Effectiveness of a preventive educational programme on the oral health of preschool children. Promot Educ. 2007; $14: 155-8$.

\section{Como citar este artigo:}

Martins TR, Juliano Y, Ribeiro AP, Ribeiro CCF, Novo NF. Influência da idade e gênero na capacidade de crianças em incorporar hábitos de saúde bucal após atividades educativas nas escolas. Rev. Aten. Saúde. 2021; 19(69): 261-269. 